\title{
Glandular Gynecomastia Excision Through Axillary Incision Following Ultra-Sound Assisted Liposuction (UAL) of the Breast
}

\author{
SHREIF MAHER, M.D.; WALEED AL-DABAANY, M.D. and AYMAN ABD EL-HAMED, M.D. \\ The Department of Plastic Surgery, Faculty of Medicine, Fayoum University, Fayoum, Egypt
}

\begin{abstract}
Background: Gynaecomastia is a medical term meaning benign enlargement of the male breast due to a physiological or pathological factor. With a reported prevalence of up to $36 \%$. Male gynaecomastia can be pure, fatty, or mixed. Simon in 1973 grouped the patients into categories according to the size of the gynaecomastia and classified gynecomastia into grades. Rohrich classified gynecomastia according to the degree of hypertrophy and put different treatment options and in cases with more than $500 \mathrm{gm}$ hypertrophy he suggested excision and liposuction. The use of ultrasound for cosmetic surgery has been introduced since the late 1980. UAL delivers ultrasonic energy directly to deep fat deposits through very tiny incisions in the skin. This technique enhances the current procedure in liposuction. Following the liposuction excision can be done via different approaches to remove the remaining glandular component of the breast.
\end{abstract}

Material and Methods: 28 male patients with gynecomastia were presented in this study, through the period from February 2012 to October 2016, in Fayoum university hospital. First full detailed history and lab examination was done. The surgery was done either under local anaesthesia with sedation or under general anaesthesia .Liposuction was done using UAL first through anterior axillary incision, and then dissection of the gland was done using the Laparoscopic Scissors. Excision of gland was done through the same incision of liposuction at the axilla. Pressure garments were applied for 4 weeks following the surgery.

Results: 26 patients (92.8\%) showed good satisfactory results in follow-up period ranging from 6 months to at least up to 2 years. 20 patients $(71.4 \%)$ had been operated under general anaesthesia and 8 patients $(28.6 \%)$ under local anaesthesia. The operative time ranged from 1 hour and 15 minutes to 2 hours with mean operative time 1 hour and 35 minutes. All the cases were done as a day cases. None of the patients showed hematomas or seromas or wound infection, one patient had areola injury healed spontaneously and one patient had depressed saucer dish deformity.

Conclusion: The UAL and axillary excision of gynecomastia has excellent results with few complications and safe procedure and aids to decreasing the skin laxity following the surgery.

Key Words: Axillary excision - Gynecomastia - Ultrasoundassisted liposuction.

\section{INTRODUCTION}

Gynecomastia is a medical term meaning benign enlargement of the male breast due to a physiological or pathological factor [1]. Gynecomastia is defined as benign, excessive breast development in male individuals. Initially thought to be a rare condition, with a reported incidence of 8 in 100,000 during World War II [2], more recent study a reported prevalence of up to $36 \%$ even as high as $40 \%$ in autopsy series [3].

Gynaecomastia is one of the most common causes for seeking medical advice for a breast condition especially in young men. Enlarged breasts can cause anxiety, and embarrassment, and social and psychological discomfort.

Gynecomastia was classified by Webster in the 1930s into the following three categories: Type one was glandular, type two was fatty glandular, and type three was simple fatty [2].

Simon in 1973 grouped the patients into categories according to the size of the gynaecomastia and classified gynecomastia into four groups [4].

- I: Minor breast enlargement without skin redundancy.

- IIa: Moderate breast enlargement without skin redundancy.

- IIb: Moderate breast enlargement with minor skin redundancy.

- III: Gross breast enlargement with skin redundancy that mimics female breast ptosis.

Bannayan and Hajdu described types of gynecomastia as florid, fibrous, and Intermediate if gynecomastia is in its fibrous phase, or present for more than 1 year, it is unlikely to regress. In such circumstances, surgery is the best option for cosmetic improvement [5]. 
Rohrich classified gynecomastia according to the degree of hypertrophy and put different treatment options [6].

In the beginning Illouz [7] used the traditional liposuction (Suction Assisted Liposuction) then the power assisted liposuction was introduced, then the ultrasound-assisted liposuction (UAL).

In 2003 Rohrich et al. [8] introduced a stepwise approach employing a new classification system using ultrasound-assisted liposuction (UAL); (Table 1).

Table (1): Classification and management of gynecomastia.

\begin{tabular}{|c|c|}
\hline Classification & Management \\
\hline \multicolumn{2}{|l|}{$\begin{array}{l}\text { Grade I, minimal hypertrophy } \\
(<250 \mathrm{~g}) \text { without ptosis: }\end{array}$} \\
\hline Primarily glandular & $\mathrm{UAL} \pm$ direct excision \\
\hline Primarily fibrous & $\mathrm{UAL} \pm$ direct excision \\
\hline \multicolumn{2}{|l|}{$\begin{array}{l}\text { Grade II, moderate hypertrophy } \\
(250-500 \mathrm{~g}) \text { without ptosis: }\end{array}$} \\
\hline Primarily glandular & $\mathrm{UAL} \pm$ direct excision \\
\hline Primarily fibrous & $\mathrm{UAL} \pm$ direct excision \\
\hline $\begin{array}{l}\text { Grade III, severe hypertrophy } \\
(>500 \mathrm{~g}) \text { with grade I ptosis }\end{array}$ & $\begin{array}{r}\text { UAL, direct excision } \pm \\
\text { delayed skin resection }\end{array}$ \\
\hline $\begin{array}{l}\text { Grade IV, severe hypertrophy } \\
(>500 \mathrm{~g}) \text { with grade II or III ptosis }\end{array}$ & $\begin{array}{r}\text { UAL, direct excision } \pm \\
\text { delayed skin resection }\end{array}$ \\
\hline
\end{tabular}

The use of ultrasound for cosmetic surgery has been introduced since the late 1980's; UAL delivers ultrasonic energy directly to deep fat deposits through very tiny incisions in the skin. This technique enhances the current procedure in liposuction [6].

UAL has several advantages in the treatment of gynecomastia. UAL breaks up the dense fibro connective tissue of the male breast more efficiently than suction-assisted lipectomy (SAL), and at higher energy settings, UAL has the capacity to remove the dense parenchymal tissue that SAL leaves behind [8].

Many techniques are available for surgical correction of gynaecomastia. Surgery is planned depending on the grade and histopathology of gynecomastia. Webster's intraareolar incision, periareolar or circumareolar incisions, Letterman's technique, and suction-assisted lipectomy are commonly used in the treatment of grades 1 and 2A gynecomastia. Superiorly or inferiorly based pedicle areolar flaps and free nipple techniques are preferred for grades $2 \mathrm{~B}$ and 3 gynaecomastia [9]. Other approaches to remove the gland surgically is through tans the nipple and through axillary incision.
In 2016 Steven et al., published a retrospective chart review including all patients who were treated, from 2000 to 2013 by the senior author, for evolution of gynecomastia management using direct pull-through excision with ultrasound-assisted liposuction from infra mammary incision [10].

The purpose of this study is to evaluate the excision of gynecomastia disc from the axillary incision after UAL, regarding the results and complications.

\section{MATERIAL AND METHODS}

The study was conducted in Fayoum University Hospital in the period between February 2012 \& October 2016.

28 patients with glandular gynaecomastia were operated upon including all degrees of gynecomastia except Simon grade III, patients who need skin excision. The age of the patient ranged between 20 and 38 years with Mean Age 28 years. Full history was taken from the patients especially drug administration, full examination was done. All patients were investigated by, $\mathrm{CBC}$, liver and renal function, prothrombin time, partial prothrombin time, INR. Some patients investigated for free testosterone, FSH thyroid function tests. Informed consent was obtained from all patients.

All the cases were photographed preoperatively. The patient was marked in the standing position, marking the outline of the disc, and the infra mammary fold and the breast outlining, then the liposuction stab incision is marked at the level of infra mammary fold at the anterior axillary line (Fig. 1).

20 patients $(71.4 \%)$ had been operated under general anaesthesia and 8 patients $(28.6 \%)$ under local anaesthesia and positioned supine with the arms abducted. Prophylactic antibiotic was given preoperatively. Sterilization by Betadine from the clavicle down to the umbilicus.

\section{The liposuction procedure:}

Infiltration: In cases with general anaesthesia both superficial and deep infiltration were performed using a standard solution of $1000 \mathrm{~mL}$ of normal saline and 1 ampoule of epinephrine 1:1000, $50 \mathrm{ml}$ xylocaine $2 \%$. With symmetric volumes infiltrated on each side [11]. In case of operating under local anaesthesia and sedation the breast tissue is infiltrated by haemostatic solution formed from saline-adrenaline 1; 500.000 and $50 \mathrm{ml}$ xylocaine $2 \%$. Each breast is infiltrated with the solution 1:1 ratio of infiltrate to estimated aspirate. 
The amount of infiltrate ranged from $300 \mathrm{cc}$ to 1000 cc. It was done through the incision using $2 \mathrm{~mm}$ multi-hole infiltration cannula then waiting for 15 minutes before liposuction to allow adrenaline and xylocaine for acting.

After completing the infiltration, a skin port protector is applied to protect the skin at the incision from burn by the Ultra sound device cannula.

Emulsification: Emulsification was performed in accordance with the chart of volume infiltrated, in the same order as the infiltration. The duration of Ultrasound treatment was 1 minute per each $100 \mathrm{~mL}$ of infiltration, but the clinical endpoint was the loss of resistance [11]. Usually we used the 3.7 probe for the breast in all cases. Continuous mode was performed and we placed the power setting at $60 \%$.

The average time for emulsification ranged between 3 to 10 minutes for each side.

Liposuction: Using a $3 \mathrm{~mm}, 4 \mathrm{~mm}$ or a $5 \mathrm{~mm}$ round-tip Mercedes cannula for the initial suction using the palm down and pinch techniques. The final contouring was performed and changes were constantly monitored by direct observation [12]. The amount of liposuction from each side ranged from $200 \mathrm{cc}$ to $1100 \mathrm{cc}$.

\section{Gland excision:}

After evacuation is complete a long laparoscopic scissor is introduced through the liposuction incision to dissect the gland from the skin leaving thin layer under the skin and relatively thicker layer below the NAC. (Fig. 2).

A Kocher clamp is introduced through the stab incision and the residual subareolar dense tissue is grasped with a clamp pulled through the incision (Fig. 3), and directly excised.

Once the appropriate contour is achieved, the lateral inframammary incisions are left open to drain any collected fluid and to avoid the need of drains. Compression garment is then used for 4 weeks.

Patients were followed after 3 days, 1 week, 4 weeks, 3 months and at least 6 months, evaluating the aesthetic results, pain, oedema and presence of complications.

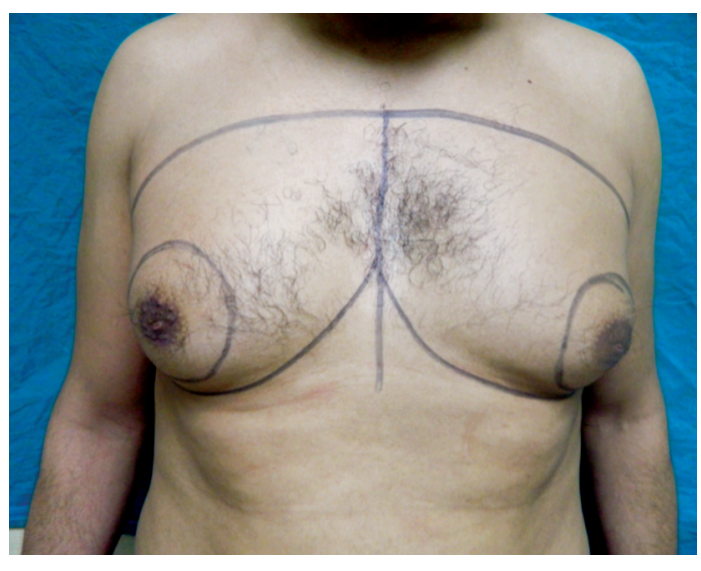

Fig. (1): Preoperative marking.

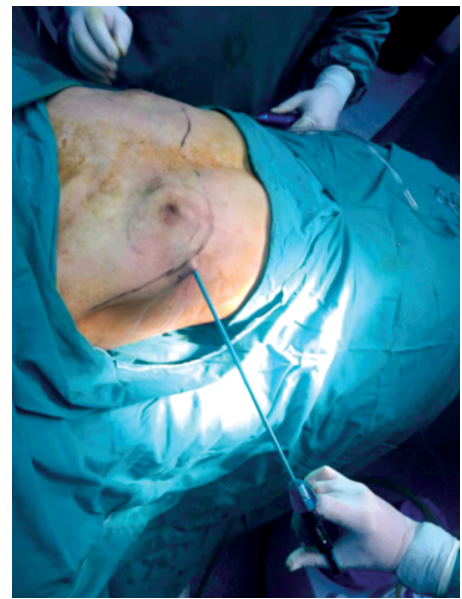

Fig. (2): Laparoscopic scissor is introduced through the liposuction incision.

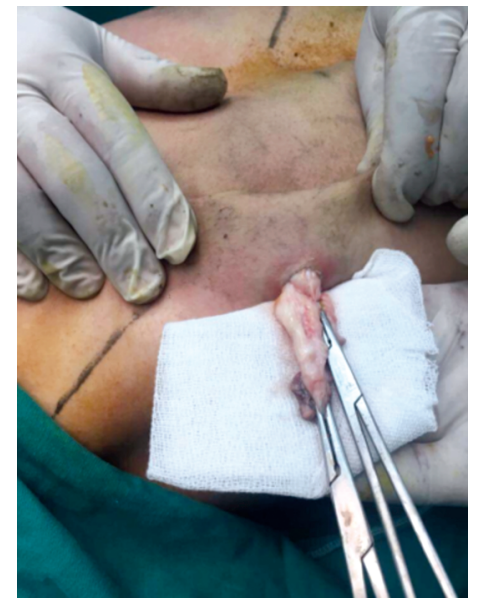

Fig. (3): The residual subareolar dense tissue is pulled through the liposuction incision.

\section{RESULTS}

During the study period (from February 2012 to October 2016) 28 male patients were enrolled in this study. Age ranged from 20 to 38 years with (mean age 28 years).

All the cases had true gynecomastia, 26 patients $(92.8 \%)$ showed good satisfactory results to the patients and the surgeons in follow-up period ranging from 6 months at least up to 2 years. The results were evaluated by the patients and the surgeons including the shape of the breast and the post-operative scar and shape of NAC and condition of the skin, and the patient and surgeons gave a score to each point from 1 to 10 (total 40 points), and overall score more than $80 \%$ (32 points) was considered good satisfactory results. It is worth saying that a considerable skin tightening was noted in the patients following the use of UAL due to the effect of ultrasound on the skin tightening. 
20 patients $(71.4 \%)$ had been operated under general anaesthesia and 8 patients (28.6\%) under local anaesthesia with light sedation.

The operative time ranged from 1 hour and 15 minutes to 2 hours with mean operative time 1 hour and 35 minutes including the time needed for infiltration and waiting for the action of adrenaline (10 to 15 minutes) and application of the Ultrasound (between 3 to 10 minutes/side according to amount of filtration 1 minute $/ 100 \mathrm{ml}$ of infiltration) and liposuction and removal of the gland.
The amount of liposuction ranged from 250cc to $1000 \mathrm{cc} / \mathrm{side}$. The wound was closed without drain insertion.

All the cases were done as a day cases. None of the patients showed hematomas or seromas or wound infection, one patient had areola injury healed spontaneously and one patient had depressed saucer dish deformity needed later fat injection, 2 patients had loss of sensation in the nipple and areola. (Figs. 4,5,6).

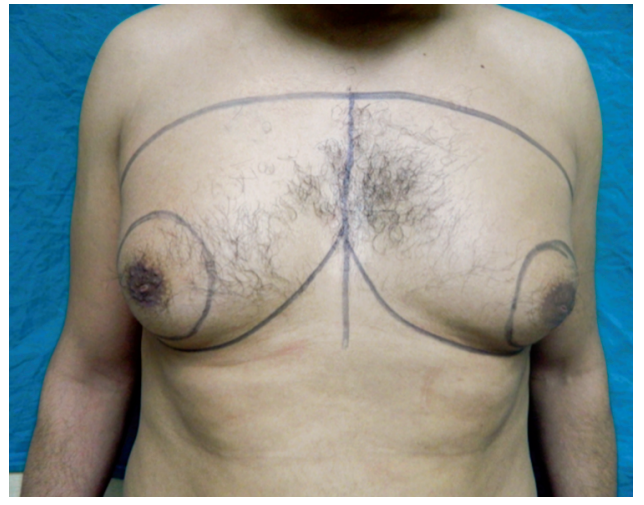

Fig. (4A): Preoperative front view.

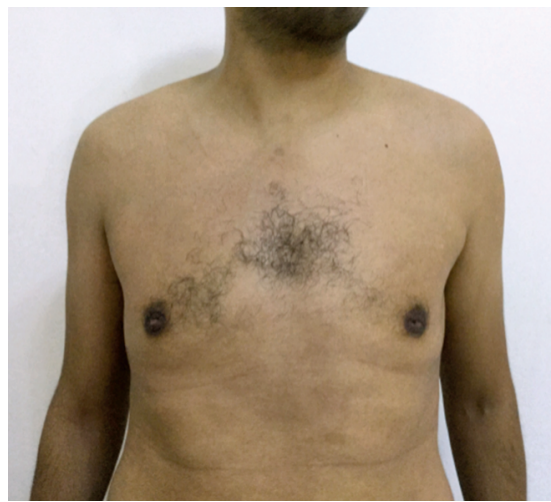

Fig. (4D): Two years postoperative front view.

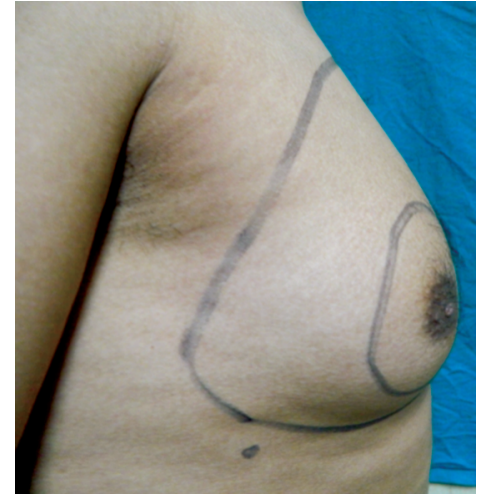

Fig. (4B): Preoperative Rt. lateral view.

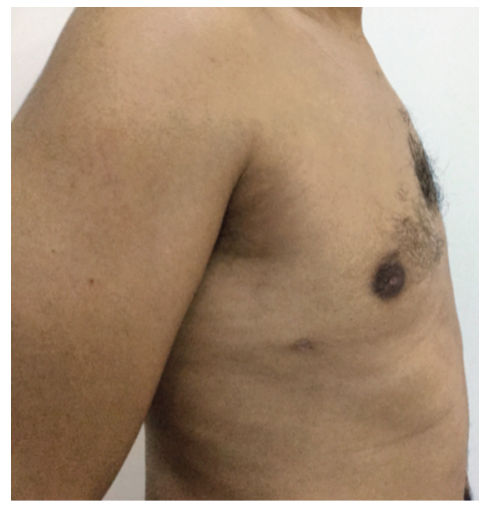

Fig. (4E): Two years postoperative Rt. lateral view.

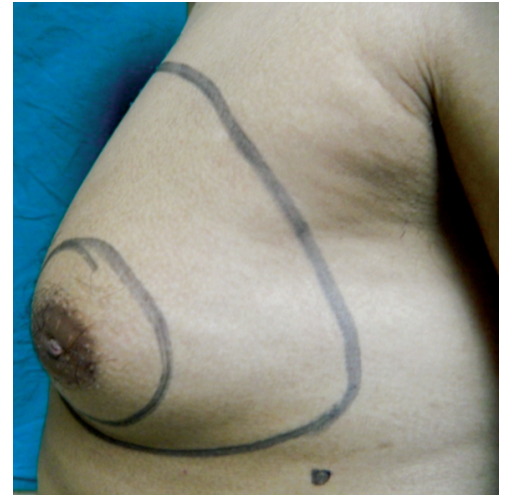

Fig. (4C): Preoperative Lt. lateral view.

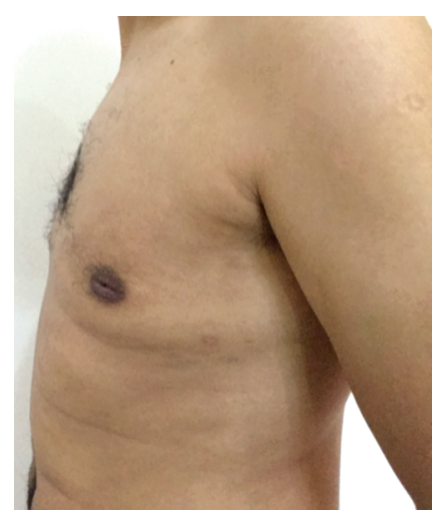

Fig. (4F): Two years postoperative Lt. lateral view.

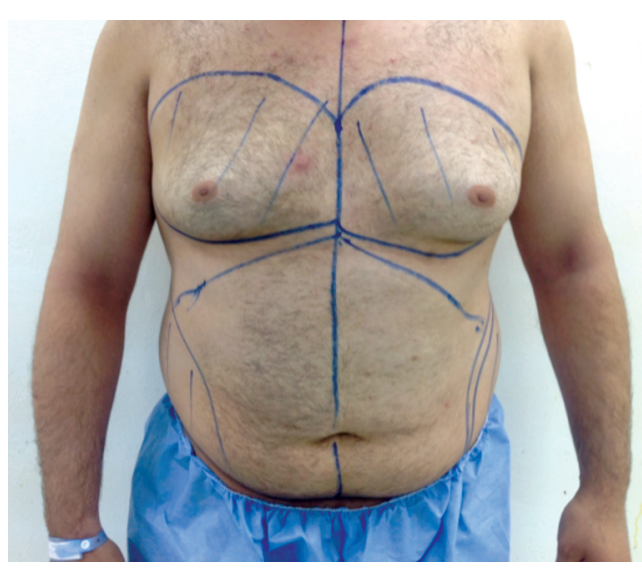

Fig. (5A): Preoperative front view.

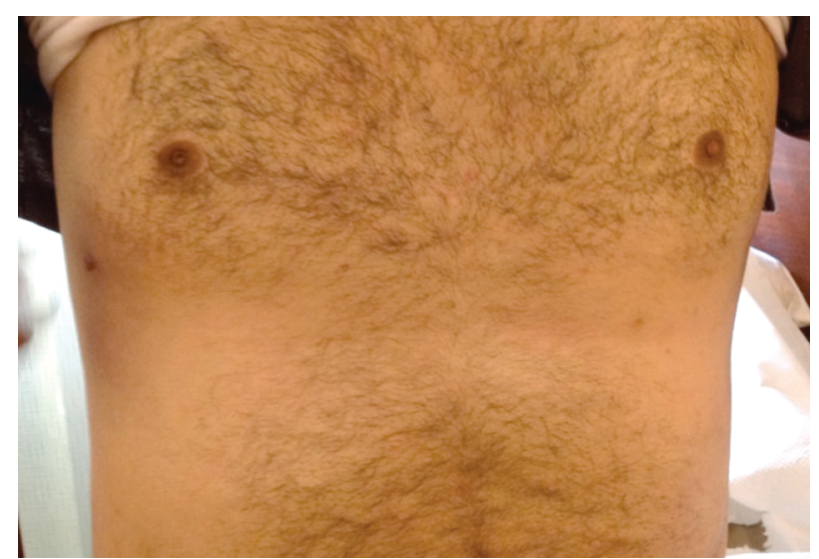

Fig. (5B): Six months post-operative front view. 


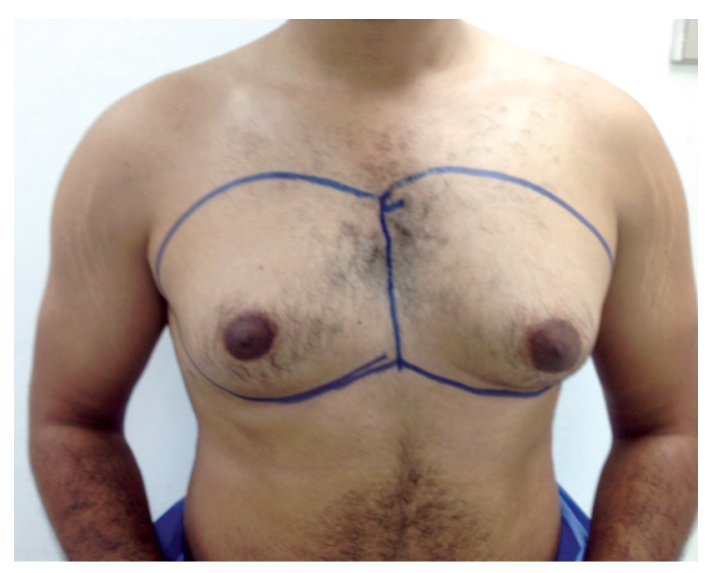

Fig. (6A): Preoperative front view.

\section{DISCUSSION}

The understanding gynecomastia continues to slowly expand. Despite the advances in medical treatment of gynecomastia, the surgical treatment remains the only effective treatment for patients with fibrous gynecomastia [13].

Webster [14] described an intra-areolar incision for gynecomastia excision. Then to minimize the appearance of the incision, others have masked the scar either by placing the incision in the axilla or by using a circumareolar incision, but the scar was sometimes visible and with distortion and depression of the NAC in some cases [14].

UAL has become widely accepted as the treatment for gynecomastia [15]. UAL gives many advantages as minimal scarring and removal of both fatty and glandular breast tissue. A considerable amount of skin retraction is obtained in patients with grade III and IV gynecomastia and sometimes obviates the need for additional procedures to remove excess skin. Recently, there have been reports of combining UAL and surgical excision of the gland within the same setting [10]. Due to the difficulty to remove the gland totally just by liposuction. But after the use of UAL it becomes easy to dissect the remaining fibres connecting the gland to the skin and the muscle by using fine instrument like the laparoscopic scissors to remove the gland from a tiny incision of the liposuction incision.

Bracaglia et al. [15], combined suction-assisted lipectomy and the pull through technique using an inframammary crease incision and an incision overlying the sternum but there was no improvement of the skin and some cosmetic problems regarding the scar on the sternum.

Mentz et al., described another technique for management of gynecomastia through a single

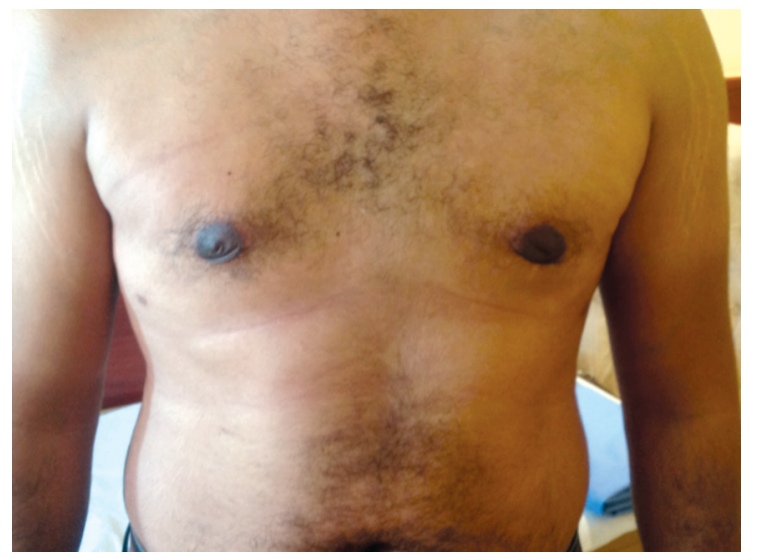

Fig. (6B): Six months post-operative front view.

incision at the 6-o'clock position of the areola with a separate incision for liposuction at the anterior axillary fold but having the problems of the scar around the areola.

Petty et al. [17] used UAL and the arthroscopic shaver to remove the subareolar fibrous component.

Morselli and Morellin et al. [16], published their experience in the pull-through technique, but with the use of 2 separate incisions to remove the gland.

Hady [12] published the Liposuction excision of gynecomastia through an axillary incision with the traditional liposuction, but with incidences of seroma and hematoma although he used suction drain at the end of operation which was not needed in our technique with the use of UAL and leaving the incision without closure.

Steven et al., published their experience with 75 patients from 2003-2010 using UAL with direct pull through using a surgical scissor from an inframammary incision they advocated grade-directed treatment, but updated their treatment algorithm to include UAL and direct excision via the pullthough technique obviating the need for a periareolar incision [10].

In our technique the use of UAL obviates the need of drains with no incidence of hematoma or seroma and provides smooth easy excision of the gland. The hidden small incision is cosmetically good for the patient. The use of laparoscopic scissors to dissect the remaining fibrous tissues provides easy access without need to enlarge the incision to introduce and open the blades of the surgical scissors.

\section{Conclusion:}

The technique of axillary excision of glandular gynecomastia following the use of UAL is safe 
effective associated with good aesthetic results with minimal incision and fast recovery and high tolerance and low complication rate, but needs more experience and more time consuming and more advanced machines.

\section{Conflicts of interest:}

The authors declare that there are no conflicts of interest or financial affiliations specific to this study.

\section{Financial support and sponsorship:}

Nil.

\section{REFERENCES}

1- Steel S.R., Martin M.J. and Place R.J.: gynaecomastia: Complications of the subcutaneous mastectomy. Am. Surg. Feb., 68 (2): 210-3, 2002.

2- Webster G.V.: Gynecomastia in the Navy. The Military Surgeon November, 1944

3- Williams M.J.: Gynecomastia: Its incidence, recognition and host characterization in 447 autopsy cases. Am. J. Med., 34: 103, 1963.

4- Simon B.E., Hoffman S. and Kahn S.: Classification and surgical correction of gynecomastia. Plast. Reconstr. Surg., 51: 48-52, 1973.

5- Bannayan G.A. and Hajdu S.I.: Gynecomastia: Clinicopathologic study of 351 cases. Am. J. Clin. Pathol., 57: 431-437, 1972.

6- Rohrich R.J., Beran S.J. and Kenkel J.M.: Ultrasound Assisted Liposuction. St. Louis: Quality Medical Publishing, 1998.

7- Illouz Y.G.: Body contouring by lipolysis: A 5-year experience with over 3000 cases. Plast. Reconstr. Surg., 72: 591-597, 1983.
8- Rohrich R.J., Ha R.Y. and Kenkel J.M.: Classification and management of gynecomastia: Defining the role of ultrasound-assisted liposuction. Plast. Reconstr. Surg., 111: 909-923, 2003

9- Celebioglu S., Ertas N.M., Ozdil K. and Oktem F.: Gynecomastia treatment with subareolar glandular pedicle. Aesth.Plast. Surg., 28: 281-286, 2004.

10- Steven H. Bailey, Dax Guenther, Fadi Constantine and Rod J. Rohrich: Gynecomastia Management: An Evolution and Refinement in Technique at UT South western Medical Centre. Plast. Reconstr. Surg. Glob Open. Jun., 4 (6): e734, 2016.

11- Alfredo E. Hoyos and John A. Millard: VASER-assisted high-definition liposculpture, Aesthetic Surgery Journal, 27 (6): 594-604, 2007.

12- Hady S. Abou Ashour: Liposuction excision of gynecomastia through an axillary liposuction opening: A novel technique. The Egyptian Journal of Surgery, 34: 170-176, 2015.

13- Braunstein G.D.: Gynecomastia. N. Engl. J. Med., 357: 1229-1237, 2007.

14- Davidson B.A.: Concentric circle operation for massive gynecomastia to excise the redundant skin. Plast. Reconstr. Surg., 63 (3), 1979.

15- Bracaglia R., Fortunato R., Gentileschi S., Seccia A. and Farallo E.: Our experience with the so-called pull-through technique combined with liposuction for management of gynecomastia. Ann. Plast. Surg., 53: 22-26, 2004.

16- Morselli P.G.: A Morellin Breast reshaping in gynecomastia by the "pull-through technique": Considerations after 15 years. Eur. J. Plast. Surg., 35: 365-371, 2012.

17- Petty P.M., Solomon M., Buchel E.W., et al.: Gynecomastia: Evolving paradigm of management and comparison of techniques. Plast. Reconstr. Surg., 125: 1301-1308, 2010. 